\title{
The signature of inhibition in theory of mind: children's predictions of behavior based on avoidance desire
}

\author{
Adam R. Petrashek • Ori Friedman
}

Published online: 16 November 2010

(C) Psychonomic Society, Inc. 2010

\begin{abstract}
Theory-of-mind reasoning may require inhibitory processing, a view for which we provide new, more direct, evidence. Our research capitalizes on the lingering property of inhibition: After a response is inhibited, it temporarily becomes more difficult to select than uninhibited responses. In two experiments, 3-year-olds predicted to which of three buckets a girl would go, given a desire to avoid one (e.g., the red one). When next predicting where a boy would play, children were biased against the location the girl had avoided. However, predictions for the boy were unbiased when not preceded by predictions for the girl. These findings suggest that preschoolers predict behavior based on avoidance desires by inhibiting the target to be avoided, as well as that the inhibition lingers, reducing the target's accessibility for subsequent predictions.
\end{abstract}

Keywords Theory-of-mind Inhibition · Avoidance desire · Cognitive development $\cdot$ Children

Reasoning about beliefs, desires, and other mental states, an ability often called "theory of mind," is crucial for predicting and understanding behavior. Theory-of-mind reasoning improves dramatically during childhood. Whereas children have difficulty on many theory-of-mind tasks at age 3, they succeed on most by age 5 (Cassidy, 1998; Leslie, German, \& Polizzi, 2005; Wellman, Cross, \& Watson, 2001). It is now widely believed that inhibitory processing is responsible, at least in part, for many of these age-related improvements in performance. This view assumes that

A. R. Petrashek $\cdot$ O. Friedman $(\square)$

Department of Psychology, University of Waterloo,

200 University Avenue W,

Waterloo, ON, Canada N2L 3G1

e-mail: friedman@uwaterloo.ca inhibitory processing improves in the preschool years (Diamond, 2006) and that such improvements allow children to meet the inhibitory demands required for certain kinds of theory-of-mind reasoning.

Two types of theory-of-mind reasoning that may require inhibitory processing are predicting behavior based on false beliefs and avoidance desires (Leslie \& Polizzi, 1998). For instance, suppose a girl can play in one of three locations but wants to avoid one. Predicting how she will behave on the basis of this avoidance desire may require first identifying the location she wants to avoid, and then inhibiting it so that a different location can be chosen as her likely target.

Two kinds of evidence lend support to this inhibitory account. First, theory-of-mind tasks can be made easier or more difficult via task manipulations (see, e.g., Mitchell \& Lacohee, 1991; Siegal \& Beattie, 1991; Yazdi, German, Defeyter, \& Siegal, 2006; Zaitchik, 1991). Many of these manipulations are thought to operate by modifying the inhibitory demands of theory-of-mind tasks, suggesting that children's difficulty with these tasks depends on the inhibitory demands they make. For instance, when inhibitory resources are scarce, both children (see, e.g., Leslie et al., 2005) and adults (German \& Hehman, 2006) have more difficulty predicting behavior for characters with avoidance desires than for characters with approach desires. Predicting the behavior of a character with a false belief is thought to tax 4-year-olds' inhibitory resources (Roth \& Leslie, 1998). Most 4-year-olds can make these predictions in the context of approach desire: They can predict that a girl who believes a marble is in a basket will look for it there, even if they know the marble is really in a box (Wellman et al., 2001). But they often fail if the girl wants to avoid the location with the marble (Cassidy, 1998; Leslie \& Polizzi, 1998). This drop in performance does not simply 
result because adding avoidance desire increases task complexity: Avoidance desire does not drop performance in an equally complex and difficult control task where belief attribution is thought to not require inhibition (Friedman \& Leslie, 2005).

The second kind of evidence favoring the inhibitory account comes from studies showing that children's performance on theory-of-mind tasks is correlated with their performance on tasks assessing inhibition (e.g., Carlson \& Moses, 2001; Rakoczy, 2010). For instance, children's performance on the false-belief task is correlated with their performance on the day-night task. In this task, children are required to say "night" when shown cards depicting day, and "day" to cards depicting night (Diamond, Kirkham, \& Amso, 2002; Gerstadt, Hong, \& Diamond, 1994). Success is thought to require inhibition of the dominant response associated with the card so that the alternative response can be selected.

Both of these lines of evidence (task manipulations, correlations) only provide indirect evidence for inhibition in theory of mind. Although we find the evidence from taskmanipulation studies compelling, it remains possible that some processing factor other than inhibition is responsible for the changes that occur with task manipulations. The evidence from correlational studies is far weaker. First, rather than indicating that theory of mind draws on inhibition, the correlations might arise instead because inhibitory processing is necessary for mental-state concepts to emerge (Moses, 2001). Second, it is an open question whether tasks designed to measure inhibition actually do so. In most of the "inhibitory" tasks used in correlational studies, the need for inhibitory processing is inferred from children's difficulty in following rules that seem likely to conflict with natural or practiced tendencies (e.g., saying "night" to day cards). However, these tasks are open to noninhibitory explanations as well (for a broader discussion of the difficulty of establishing that a task requires inhibitory processing, see MacLeod, Dodd, Sheard, Wilson, \& Bibi, 2003).

The research presented here aims to provide more decisive evidence that inhibitory processing is involved in children's theory-of-mind reasoning. Our research capitalizes on the lingering property of inhibition: Once a response is inhibited, the inhibition lingers, making the response more difficult to select than uninhibited responses. Although diverse inhibitory accounts have appealed to the lingering property of inhibition (e.g., Mayr \& Keele, 2000; Posner \& Cohen, 1984), this signature of inhibition has never been found in the context of theory of mind.

In two experiments, we looked for evidence of lingering inhibition in theory of mind. We investigated whether lingering inhibition arises when 3-year-olds predict behavior based on an avoidance desire. Three-year-olds were investigated because they sometimes fail to predict behavior based on avoidance desires in very simple tasks (Leslie et al., 2005). According to the inhibitory account, these occasional failures result because 3 -year-olds are challenged in meeting the inhibitory demands of making such predictions. Thus, it seemed especially likely that predicting behavior based on avoidance desire would have lingering consequences for 3-year-olds.

\section{Experiment 1}

Method

Participants Three-year-olds were randomly assigned to one of two conditions, double prediction or single prediction. Sixty-three children successfully completed the task: 29 in the double-prediction condition (14 girls; age range = $3 ; 0$ [i.e., 3 years 0 months] to $3 ; 11, M=3 ; 7$ ) and 34 in the single-prediction condition (19 girls; age range $=3 ; 1$ to $3 ; 11, M=3 ; 6)$. Another 5 children were tested in the double-prediction condition but were excluded for not completing the task successfully. These children did not succeed in predicting behavior based on avoidance desires, so their responses are not useful for assessing whether lingering inhibition results from making such predictions.

Participants were recruited from, and tested at, their daycares and preschools. Most participants were Caucasian and from middle-class families, though demographic information was not formally collected.

Materials and procedure Children watched a scenario enacted on a foam board stage using three large plastic buckets (red, yellow, blue) lined in a row and small toy replicas of a boy and a girl. Children were told that the characters each want to play under a bucket, though not under the same bucket as each other, and that the girl wants to avoid one of the buckets (the bucket on the left or the right, counterbalanced within each condition). In the double-prediction condition, children were first asked to predict where the girl would play, and then to predict where the boy would play. In the single-prediction condition, children were told where the girl would play and then asked where the boy would play. See Table 1 for sample story scripts.

The location where the girl wanted to play in the singleprediction condition was determined by the response of the previously tested child in the double-prediction condition. Exceptions to this procedure occurred when children in the double-prediction task wrongly chose the bucket that the girl wanted to avoid; in these cases, single-prediction participants were told that the girl would go to the bucket in the middle. 
Table 1 Experiment 1: Example script from double-prediction and single-prediction conditions

We're going to play a game, and it's about these two kids. This is a girl, and this is a boy. And they like to play under these big buckets. How many buckets are there? What color is this one? And this one? And what color is this one? Now, the boy and the girl never play under the same bucket as each other. And the girl doesn't want to play under the red bucket.

Double-prediction

Which bucket will the girl play under?

Which bucket will the boy play under?

\section{Single-prediction}

So the girl will play under this bucket.

Which bucket will the boy play under?
Results and discussion

In predicting for the boy, children should not choose the location where the girl will play, but instead either the location she wants to avoid or the remaining "neutral location." Although both answers are correct, the inhibitory account predicts that children's responses should differ across conditions. In the double-prediction condition, in which children initially predicted where the girl would play, they should inhibit the location she avoids. This "avoidlocation" should remain inhibited and inaccessible when children subsequently predict for the boy, so they should be biased to choose the neutral location. This bias was not expected in the single-prediction condition. Here, children did not predict where the girl would play, so the avoidlocation should remain uninhibited and accessible.

Consistent with these expectations, children's predictions differed across conditions (Upton's $\chi^{2}, p=.036$, one-tailed; Upton, 1982): In the double-prediction condition, most children $(72.4 \%)$ predicted that the boy would go to the neutral location (Binomial, $N=29, x=8, p=.024$, twotailed) rather than the avoid-location. In the singleprediction condition, each location was chosen by exactly half of the children (Binomial, $N=34, x=17$, n.s).

Children's bias in the double-prediction condition suggests that they inhibited the avoid-location when predicting the girl's behavior. The bias would not have occurred if predicting for the girl only required children to selectively activate the location where the girl would play, without inhibiting the avoid-location. If the location chosen for the girl were strongly activated, children might be expected to have difficulty avoiding it when predicting for the boy. Even if this difficulty were overcome, the uninhibited avoid-location would be left as accessible as the neutral location, so the children would be expected to choose between these two options at chance when predicting for the boy.

The bias in the double-prediction condition could also not have resulted from children extending the girl's avoidance desire to the boy, and simply following a general rule to never choose this location. Following this rule should have led children to also prefer the neutral location in the single-prediction condition; they did not, however, and were as likely to choose the avoid-location.
However, the findings might be explained by a gradedmemory version of this "general rule" account (this account is loosely inspired by Munakata, Morton, \& Stedron, 2003, pp. 100-101). Children might adhere to the general rule ("never choose the avoid-location") when it is strongly represented in memory, but not when it has decayed. In the double-prediction condition, children should initially follow the rule when predicting for the girl. This will maintain the rule in memory, so that children follow it again when predicting for the boy, leading them to favor the neutral location over the avoid-location. In the single-prediction condition, children do not need to use the rule initially (because they are told where the girl will go). The rule thus degrades and is not followed when they predict for the boy, resulting in chance performance. To rule out this possibility, we conducted a second experiment using a task not open to this rival explanation.

\section{Experiment 2}

\section{Method}

Participants Three-year-olds were randomly assigned to one of two conditions, avoider first or avoider second. Sixty-five children successfully completed the task: 35 in the avoider-first condition (20 girls; age range $=3 ; 0$ to $3 ; 11$, $M=3 ; 6)$ and 30 in the avoider-second condition (15 girls; age range $=3 ; 0$ to $3 ; 11, M=3 ; 7$ ). Another 9 children were tested $(2$ in the avoider-first condition, 7 in the avoidersecond condition) but were excluded for not completing the task successfully. The participants were recruited from and tested at their daycares and preschools. Most were Caucasian and from middle-class families, though demographic information was not formally collected.

Materials and procedure Children were shown the same scenario as in Experiment 1, except that we counterbalanced whether it was the girl or the boy who wanted to avoid a specific location. For ease of exposition, we continue to refer to the "avoider" as the girl and to the other character as the boy. In the "standard-order" condition, children first predicted for the girl and then for the boy; this condition was identical to the double-prediction condition 
in Experiment 1. In the "reverse-order" condition, children predicted in the reverse order.

\section{Results and discussion}

As in Experiment 1, the inhibitory account forecasted distinct response patterns for children's predictions of where the boy would play. The standard-order condition was essentially identical to the double-prediction condition in Experiment 1, so children should again have been biased to choose the neutral location when predicting for the boy. This was in fact found: $67.6 \%$ of the children predicted that the boy would go to the neutral location rather than the avoidlocation (Binomial, $N=35, x=11, p=.04$, two-tailed).

In the reverse-order condition, children initially predicted where the boy would play and could choose from all three locations, rather than from two. All three locations should have been equally accessible (because children should not have applied inhibition until they predicted for the girl), so approximately one-third of the children should have chosen the avoid-location. ${ }^{1}$ This expectation was also met: $33.3 \%$ of children chose the avoid-location (Binomial, chance $=.33, N=30, x=10$, n.s.). This location was always an "end" bucket (i.e., right or left side); of the children who did not choose it, $36.7 \%$ chose the other end bucket, and $30.0 \%$ chose the middle bucket. Hence, children's selections were not influenced by a geometric bias to choose or avoid the middle bucket. Even when our analysis only included the two end buckets (chance $=.50$ ), children were still not biased against the avoid-location (Binomial, $N=21, x=10$, n.s.). These findings rule out the possibility that children followed the general rule to never choose the avoid-location, because following this rule should have biased them away from it.

These findings also rule out the graded-memory version of the general-rule account, in which children only follow the rule when it is strongly represented in memory. In the reverse-order condition, children predicted for the boy immediately after being told which bucket the girl wanted to avoid. The general rule ("never choose the avoidlocation") should have been strongly represented in memory, leading children to be biased away from that location. Again, children in the reverse-order condition did not show this bias, suggesting that they were not adhering to a general rule.

\footnotetext{
${ }^{1}$ Against this theory, one could posit that children should be biased to predict that the boy will go to the avoid-location, because this prediction guarantees that the boy will not take a location the girl might want. However, it was expected that children would not reason in this way. Such reasoning would require children to plan a future sequence (i.e., to have already planned where the girl will play when initially predicting for the boy), and 3-year-olds are poor at such planning (see, e.g., McColgan \& McCormack, 2008).
}

\section{General discussion}

After 3-year-olds predicted where a girl would go in avoiding a certain location, they were subsequently biased against the avoided location when predicting for a boy (Experiments 1 and 2). Predictions for the boy were unbiased when children did not initially predict for the girl, either because they were told where she would go (Experiment 1) or because they predicted for the boy first (Experiment 2). Children thus could not have simply decided never to choose the location the girl wanted to avoid. Instead, the findings suggest that when children predict behavior based on an avoidance desire, they inhibit the target to be avoided, leaving it less accessible for subsequent predictions.

A similar response bias was found in children's folkphysics reasoning in a modified version of Hood's (1995) tubes task. The tubes task uses an apparatus of three bent opaque tubes, which are arranged so that the upper end of each tube is directly above the bottom end of a different tube. In each trial, an object is dropped into a tube's upper end, causing the object to land in a cup at the tube's bottom end. Preschoolers often wrongly look for the object in the cup directly below the tube opening the object was dropped into, and successful search may require children to inhibit this "gravity cup." This was tested by asking children (who could successfully find the object) to predict the behavior of a character who wants to avoid the dropped object (Freeman, Hood, \& Meehan, 2004). At age 4, children were biased away from the gravity cup, suggesting that they initially inhibited it when determining the object's location, making it difficult to select when they subsequently predicted the character's behavior.

The present findings, though, are the first to provide evidence for lingering inhibition in children's theory of mind. Whereas most studies of inhibition in theory of mind have focused on children's ability to attribute false beliefs, the present experiments provide evidence that inhibition is also needed when they consider avoidance desires. Thus, our findings suggest that inhibition plays a more central part in children's theory-of-mind reasoning than is generally claimed. We believe these findings also provide the most direct evidence to date for inhibition in theory-ofmind reasoning. The findings support the "selection processing" account of inhibition in theory-of-mind development (e.g., Leslie, Friedman, \& German, 2004; Leslie \& Thaiss, 1992), which originated the claim that inhibition is often needed in predicting behavior in the context of avoidance desire. Our results are also broadly consistent with other accounts (e.g., Carlson \& Moses, 2001) that are similar to the selection processing account in claiming that children's theory-of-mind competence can be masked by poor performance resulting from limited inhibitory resources. 
As such, our findings support the view that improvements in children's theory-of-mind performance do not necessarily reflect conceptual development, but instead result from gains in their ability to meet the inhibitory demands of certain types of theory-of-mind reasoning.

Finally, in demonstrating a new response bias in children's theory of mind, the present research shows that inhibition does not just influence whether children succeed when reasoning about theory of mind. Rather, our research joins with other work (Friedman \& Leslie, 2004a, 2004b) in showing that inhibition also influences how children succeed, by swaying their choice between equally correct answers.

Author Note We thank Colin MacLeod for interesting and helpful discussions regarding the notion of inhibition. This work was funded by a grant from the Natural Sciences and Engineering Research Council of Canada awarded to O.F.

\section{References}

Carlson, S. M., \& Moses, L. J. (2001). Individual differences in inhibitory control and children's theory of mind. Child Development, 72, 1032-1053. doi:10.1111/1467-8624.00333

Cassidy, K. W. (1998). Three- and four-year-old children's ability to use desire- and belief-based reasoning. Cognition, 66, B1-B11. doi:10.1016/S0010-0277(98)00008-0

Diamond, A. (2006). The early development of executive functions. In E. Bialystok \& F. Craik (Eds.), Lifespan cognition: Mechanisms of change (pp. 70-95). New York: Oxford University Press.

Diamond, A., Kirkham, N., \& Amso, D. (2002). Conditions under which young children can hold two rules in mind and inhibit a prepotent response. Developmental Psychology, 38, 352-362. doi:10.1037/0012-1649.38.3.352

Freeman, N. H., Hood, B. M., \& Meehan, C. (2004). Young children who abandon error behaviourally still have to free themselves mentally: A retrospective test for inhibition in intuitive physics. Developmental Science, 7, 277-282. doi:10.1111/j.14677687.2004.00346.x

Friedman, O., \& Leslie, A. M. (2004a). A developmental shift in processes underlying successful belief-desire reasoning. Cognitive Science, 28, 963-977. doi:10.1207/s15516709 $\operatorname{cog} 28064$

Friedman, O., \& Leslie, A. M. (2004b). Mechanisms of belief-desire reasoning: Inhibition and bias. Psychological Science, 15, 547552. doi:10.1111/j.0956-7976.2004.00717.x

Friedman, O., \& Leslie, A. M. (2005). Processing demands in beliefdesire reasoning: Inhibition or general difficulty? Developmental Science, 8, 218-225. doi:10.1111/j.1467-7687.2005.00410.x

German, T. P., \& Hehman, J. A. (2006). Representational and executive selection resources in "theory of mind": Evidence from compromised belief-desire reasoning in old age. Cognition, 101, 129-152. doi:10.1016/j.cognition.2005.05.007

Gerstadt, C. L., Hong, Y. J., \& Diamond, A. (1994). The relationship between cognition and action: Performance of children 31/2-7 years old on a Stroop-like day-night test. Cognition, 53, 129153. doi:10.1016/0010-0277(94)90068-X
Hood, B. M. (1995). Gravity rules for 2- to 4-year olds? Cognitive Development, 10, 577-598. doi:10.1016/0885-2014(95)90027-6

Leslie, A. M., \& Polizzi, P. (1998). Inhibitory processing in the false belief task: Two conjectures. Developmental Science, 1, 247254. doi:10.1111/1467-7687.00038

Leslie, A. M., \& Thaiss, L. (1992). Domain specificity in conceptual development: Neuropsychological evidence from autism. Cognition, 43, 225-251. doi:10.1016/0010-0277(92) 90013-8

Leslie, A. M., Friedman, O., \& German, T. P. (2004). Core mechanisms in "theory of mind". Trends in Cognitive Sciences, 8, 528-533. doi:10.1016/j.tics.2004.10.001

Leslie, A. M., German, T. P., \& Polizzi, P. (2005). Belief-desire reasoning as a process of selection. Cognitive Psychology, 50, 45-85. doi:10.1016/j.cogpsych.2004.06.002

MacLeod, C. M., Dodd, M. D., Sheard, E. D., Wilson, D. E., \& Bibi, U. (2003). In opposition to inhibition. In B. H. Ross (Ed.), The psychology of learning and motivation (Vol. 43, pp. 163-214). San Diego: Academic Press.

Mayr, U., \& Keele, S. W. (2000). Changing internal constraints on action: The role of backward inhibition. Journal of Experimental Psychology: General, 129, 4-26. doi:10.1037/0096-3445.129.1.4

McColgan, K. L., \& McCormack, T. (2008). Searching and planning: Young children's reasoning about future event sequences. Child Development, 79, 1477-1497. doi:10.1111/j.1467-8624.2008. 01200.x

Mitchell, P., \& Lacohee, H. (1991). Children's early understanding of false belief. Cognition, 39, 107-127. doi:10.1016/0010-0277(91) 90040-B

Moses, L. J. (2001). Executive accounts of theory of mind development. Child Development, 72, 688-690. doi:10.1111/ 1467-8624.00306

Munakata, Y., Morton, J. B., \& Stedron, J. M. (2003). The role of prefrontal cortex in perseveration: Developmental and computational explorations. In P. Quinlan (Ed.), Connectionist models of development. Hove: Psychology Press.

Posner, M. I., \& Cohen, Y. (1984). Components of visual orienting. In H. Bouma \& D. G. Bouwhuis (Eds.), Attention and performance $X$ (pp. 531-556). Hillsdale: Erlbaum.

Rakoczy, H. (2010). Executive function and the development of belief-desire psychology. Developmental Science, 13, 648-661. doi:10.1111/j.1467-7687.2009.00922.x

Roth, D., \& Leslie, A. M. (1998). Solving belief problems: Toward a task analysis. Cognition, 66, 1-31. doi:10.1016/S0010-0277(98) 00005-5

Siegal, M., \& Beattie, K. (1991). Where to look first for children's knowledge of false beliefs. Cognition, 38, 1-12. doi:10.1016/ 0010-0277(91)90020-5

Upton, G. J. G. (1982). A comparison of alternative tests for the $2 \times 2$ comparative trial. Journal of the Royal Statistical Society: Series A, 145, 86-105.

Wellman, H. M., Cross, D., \& Watson, J. (2001). Meta-analysis of theory-of-mind development: The truth about false belief. Child Development, 72, 655-684. doi:10.1111/1467-8624.00304

Yazdi, A. A., German, T. P., Defeyter, M. A., \& Siegal, M. (2006). Competence and performance in belief-desire reasoning across two cultures: The truth, the whole truth and nothing but the truth about false belief? Cognition, 100, 343-368. doi:10.1016/j. cognition.2005.05.004

Zaitchik, D. (1991). Is only seeing really believing? Sources of the true belief in the false belief task. Cognitive Development, 6, 91-103. 